\title{
Glossotonsillar Sulcus
}

National Cancer Institute

\section{Source}

National Cancer Institute. Glossotonsillar Sulcus. NCI Thesaurus. Code C129815.

The bottom part of the palatine tonsil where it blends into the lingual tonsil tissue. 